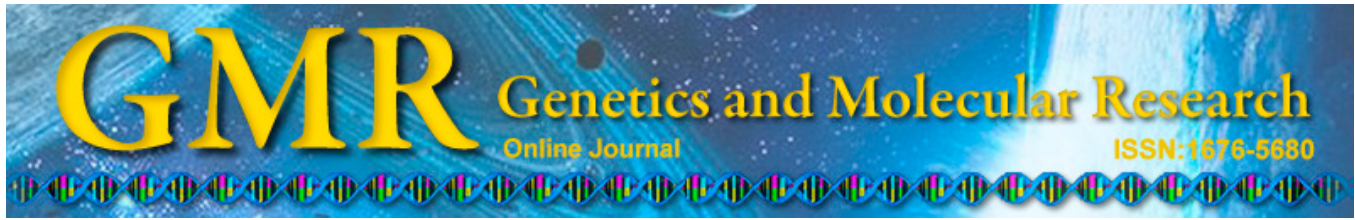

\title{
Identification of quantitative trait loci conferring blast resistance in Bodao, a japonica rice landrace
}

\author{
J. Huan*, Y.M. Bao*, Y.Y. Wu, G.Y. Zeng, W.W. He, L.L. Dang, \\ J.F. Wang and H.S. Zhang \\ State Key Laboratory of Crop Genetics and Germplasm Enhancement, \\ College of Agriculture, Nanjing Agricultural University, Nanjing, China \\ *These authors contributed equally to this study. \\ Corresponding author: H.S. Zhang \\ E-mail: hszhang@njau.edu.cn
}

Genet. Mol. Res. 13 (4): 9756-9765 (2014)

Received March 26, 2014

Accepted July 19, 2014

Published November 27, 2014

DOI http://dx.doi.org/10.4238/2014.November.27.3

\begin{abstract}
Bodao, a japonica landrace from the Taihu Lake region of China, is highly resistant to most Chinese isolates of Magnaporthe oryzea, a form of rice blast. To effectively dissect the influence of genetics on this blast resistance, a population of 155 recombinant inbred lines $\left(\mathrm{F}_{2: 8}\right)$ derived from a cross of Bodao x Suyunuo was inoculated with 12 blast isolates. Using a quantitative trait locus (QTL) mapping approach, 13 QTL on chromosomes 1, 2, 9, 11, and 12 were detected from Bodao. Five QTL, including qtl11-1-1, qtl11-3-7, qtl11-4-9, qtl12-1-1, and qtl12-2-3, have not been previously reported. The qtl11-3-7 and qtl11-4-9 may be the two main effective QTL and resistant to 7 and 9 isolates, respectively. The results of the present study will be valuable for the fine mapping and cloning of these two new resistance genes.
\end{abstract}

Key words: Rice; Blast; Recombinant inbred lines; Blast resistance; QTL mapping 


\section{INTRODUCTION}

Rice blast, which is caused by the fungal pathogen Magnaporthe oryzae, is one of the most devastating diseases affecting rice and severely limits the stability of rice production worldwide. Application of rice varieties with broad-spectrum resistance to pathogens has been the most effective and economical strategy for controlling disease (Hulbert et al., 2001). Because of the genetic instability and pathogenic variability of $M$. oryzae, host resistance generally decreases after a few years of intensive agriculture (Ou, 1979; Dean et al., 2005). Therefore, to breed blast resistance in rice, more durable resistance genes or quantitative trait loci (QTL) should be identified and pyramided through marker-aided selection (Marcel et al., 2007).

Blast resistance in rice is generally classified into 2 main types: complete resistance and partial resistance (Parlevliet, 1979). Complete resistance is race-specific and is controlled by a single-resistance gene, which can be recognized by cognate avirulence (Avr) genes. In contrast, partial resistance is controlled by QTL (Vergne et al., 2010). Over the past several years, more than 100 resistance genes or QTL have been identified (Liu et al., 2010). With the exception of chromosome 3, these genes are distributed on 11 of 12 rice chromosomes. Most are dominant, except for the recessive gene pi21 (Jeung et al., 2007). Thus far, at least 20 resistance (R) genes have been cloned (Bryan et al., 2000; Liu et al., 2010; Okuyama et al., 2011; Rai et al., 2011; Yuan et al., 2011; Zhai et al., 2011; Hua et al., 2012), many of which have been organized into gene clusters, such as Pi2 loci (Pi2/ Piz-t/Pi9) (Deng et al., 2006; Qu et al., 2006; Zhou et al., 2006) and Pik loci (Pik/Pik-m/ Pik-p/Pi1) (Ashikawa et al., 2008; Yuan et al., 2011; Zhai et al., 2011; Hua et al., 2012). Among the 20 cloned $R$ genes, 18 genes encode nucleotide binding sites and leucine-rich repeat proteins, except for $\mathrm{Pi}-\mathrm{d} 2$, which encodes a B-lectin receptor kinase (Chen et al., 2006), and pi21, which encodes a proline-rich protein that contains a putative heavy metalbinding domain and protein-protein interaction motifs (Fukuoka et al., 2009).

In our previous study, we demonstrated that Bodao, a japonica rice landrace from the Taihu Lake region, is highly resistant to many isolates from China and Japan ( $\mathrm{Li}$ et al., 2007). In the present study, lesion scores and a simple sequence repeat (SSR) molecular linkage map were produced and resistance QTL corresponding to 11 isolates from China and 1 isolate from Japan were identified in Bodao.

\section{MATERIAL AND METHODS}

\section{Plant materials and growth}

Two landraces of japonica rice (Oryza sativa L.), Bodao and Suyunuo and 155 recombinant inbred lines (RILs; $F_{2: 8}$ ) derived from a cross of Bodao x Suyunuo were used in the present study. Bodao reportedly has a high level of resistance to most blast isolates, while Suyunuo is susceptible (Li et al., 2007). The seeds of 2 parents and RILs were sown in a plastic tray with dimensions of $60 \times 30 \times 5 \mathrm{~cm}$ as described by Wang et al. (2002). All seedlings were grown in a greenhouse $\left(28^{\circ}-30^{\circ} \mathrm{C} /\right.$ day and $20^{\circ}-22^{\circ} \mathrm{C} /$ night) and were inoculated at the 4-leaf stage with the pathogen to evaluate their resistance to blast. 


\section{Pathogens and inoculation}

Eleven isolates belonging to 7 different Chinese races, including ZA, ZB, ZC, ZD, ZE, ZF, and ZG (Ling et al., 2004), were provided by the Plant Protection Research Institute, Jiangsu Academy of Agricultural Sciences, and 1 Japanese isolate Hoku1 was provided by the Institute of Crop Sciences, Chinese Academy of Agricultural Sciences (Table 1). Isolates were grown on straw decoction and corn agar media at $28^{\circ} \mathrm{C}$ in the dark for 7 days, and then kept under continuous fluorescent light for 5 days for conidiation (Zhang et al., 2011).

\begin{tabular}{|c|c|c|}
\hline \multirow[t]{2}{*}{ Isolate (race) } & \multicolumn{2}{|c|}{ Phenotype (lesion score) ${ }^{\mathrm{a}}$} \\
\hline & Bodao & Suyunuo \\
\hline 2009-5-1(ZA7) & $\mathrm{R}(0)$ & $\mathrm{S}(5)$ \\
\hline 2009-5-2(ZA7) & $\mathrm{R}(0)$ & S (4) \\
\hline 2009-15(ZB13) & $\mathrm{R}(0)$ & S (4) \\
\hline 2009-9(ZB15) & MR (2) & S (5) \\
\hline 2009-12-3(ZC3) & $\mathrm{R}(0)$ & S (5) \\
\hline 2009-8-2(ZC13) & $\mathrm{R}(0)$ & S (5) \\
\hline 2009-13(ZD5) & $\mathrm{R}(1)$ & S (5) \\
\hline 2009-2-1(ZD7) & $\mathrm{R}(0)$ & $\mathrm{S}(5)$ \\
\hline 2009-11-1(ZE3) & $\mathrm{R}(0)$ & S (4.6) \\
\hline 2009-10(ZF1) & $\mathrm{R}(0)$ & $\mathrm{S}(5)$ \\
\hline 2009-7(ZG1) & $\mathrm{R}(0)$ & $\mathrm{S}(5)$ \\
\hline Hokul(ZG1) & $\mathrm{R}(0)$ & $S(5)$ \\
\hline
\end{tabular}

$\mathrm{R}=$ resistant; $\mathrm{MR}=$ moderately resistant; $\mathrm{S}=$ susceptible.

Seedlings at the 4-leaf stage were placed into inoculation chambers and were inoculated by spraying with a conidial suspension $\left(5 \times 10^{4}\right.$ conidial $\left./ \mathrm{mL}\right)$ with several drops of Tween-20 as described by Wang et al. (2002). Inoculated plants were kept in chambers at $28^{\circ} \mathrm{C}, 95 \%$ relative humidity, and darkness for $24 \mathrm{~h}$ and were subsequently transferred to the greenhouse for incubation at $100 \%$ relative humidity, which was achieved by intermittently spraying the seedlings with water. Each line was inoculated in 2 independent experiments, and 3 replications were performed for each experiment.

Based on the lesion type and area, a lesion score (LS) of 0-5 was recorded for each seedling 7 days after inoculation according to the referred standard (Mackill and Bonman, 1992; Shi et al., 2010). For each line, the average lesion score of 10 seedlings was used for genetic analysis.

\section{Construction of molecular linkage map}

To construct the molecular linkage map, $0.5 \mathrm{~g}$ leaves from 2 parents and 155 RILs were separately collected for DNA extraction by sodium dodecyl sulfate. A total of 1687 SSR markers referenced in the International Rice Microsatellite Initiative (IRMI, http://www. gramene.org) were used to screen polymorphisms between 2 parents and 153 SSR markers with polymorphisms between 2 parents were used to construct the map. A genetic map with a total distance of $1716.7 \mathrm{cM}$ and an average distance of $11.22 \mathrm{cM}$ between 2 markers was constructed by Mapmaker/EXP v.0.3 (Lander and Kruglyak, 1995). Recombination fractions were converted into centimorgans using Kosambi's mapping function (Kosambi, 1944). A final map was drawn using MapDraw 2.1 (Liu and Meng, 2003). 


\section{Data analysis}

QTL analysis was performed by Windows QTL Cartographer using the method of composite interval mapping (CIM; Zeng, 1994). CIM was computed using windows QTL Cartographer 2.5 (Wang et al., 2007). A limit of detection (LOD) threshold of 2.5 was used to detect QTL as described by Lander and Kruglyak (1995). In the present study, QTL were labeled $q t l A-B-C$ as described by Shi et al. (2010), where $A$ indicates the chromosome number, $B$ represents the QTL, and $C$ indicates the number of isolates corresponding to the QTL.

\section{RESULTS}

\section{Resistance of parents and RIL population to blast}

As expected, Bodao was highly resistant to 12 isolates, including 2009-5-1(ZA7), 2009-5-2(ZA7), 2009-15(ZB13), 2009-9(ZB15), 2009-12-3(ZC3), 2009-8-2(ZC13), 200913(ZD5), 2009-2-1(ZD7), 2009-11-1(ZE3), 2009-10(ZF1), 2009-7(ZG1), and Hoku1(ZG1), with LS of 0-2, while Suyunuo was highly susceptible to all isolates tested with LS of 4-5 (Table 1). The RIL population showed varying reactions to all 12 blast isolates with continuous frequency distributions and transgressive segregation in LS, indicating polygenic and quantitative resistance to blast in resistant landrace Bodao (Figure 1).

A

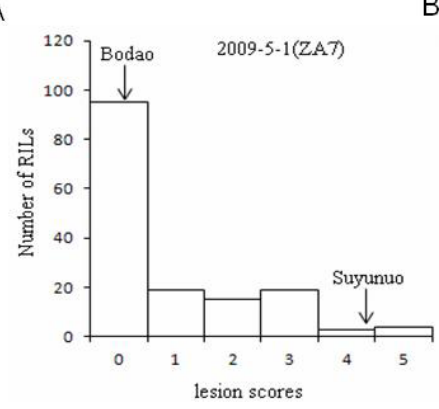

$\mathrm{D}$

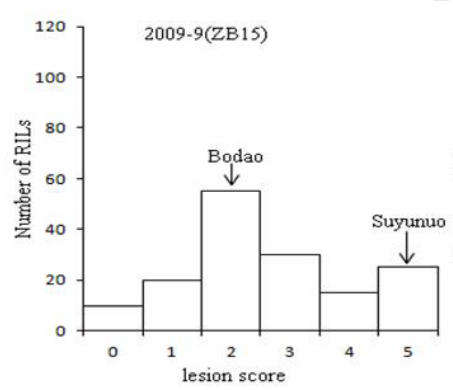

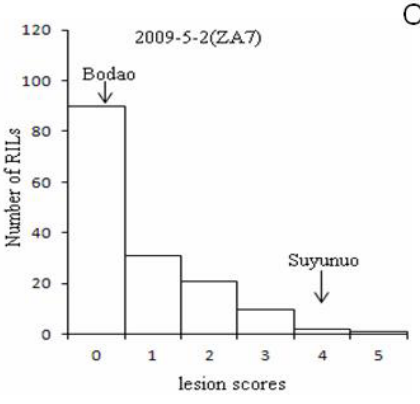

E

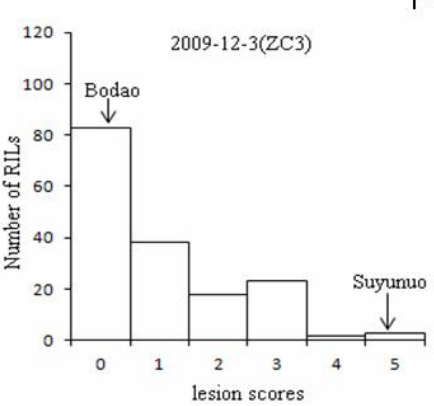

$\mathrm{F}$
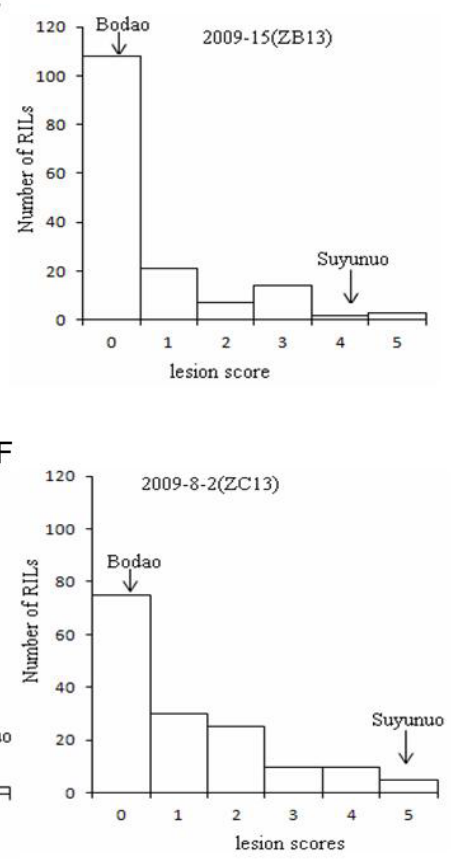

Figure 1. Distribution frequency of lesion score (LS) obtained on the progeny of 155 recombinant inbred lines (RILs) towards 12 isolates (A-M). X-axis is the LS of the lines, Y-axis is the number of lines with the same LS. 


\section{Identification of resistance loci in Bodao}

Using the CIM method, 13 QTL were detected to have resistance to 12 blast isolates (Table 2 and Figure 2). These QTL were distributed on 5 various chromosomes, including 7 QTL on chromosome 11, 2 on chromosome 9, 2 on chromosome 12, and 1 each on chromosome 1 and 2.

\begin{tabular}{|c|c|c|c|c|c|c|c|c|}
\hline QTL & Isolates & $\mathrm{Chr}^{\mathrm{a}}$ & Position $^{\mathrm{b}}(\mathrm{cM})$ & Marker left & Marker right & $\mathrm{LOD}^{\mathrm{c}}$ & Additive $^{d}$ & $\mathrm{R}^{2}(\%)^{\mathrm{e}}$ \\
\hline \multirow[t]{5}{*}{ qtll-1-5 } & 2009-11-1 & 1 & 1.20 & RM3740 & RM3652 & 7.88 & -0.80 & 11.91 \\
\hline & $2009-10$ & 1 & 1.20 & RM3740 & RM3652 & 6.47 & -0.64 & 10.77 \\
\hline & $2009-7$ & 1 & 1.20 & RM3740 & RM3652 & 4.62 & -0.41 & 13.35 \\
\hline & Hoku1 & 1 & 1.20 & RM3740 & RM3652 & 3.52 & -0.48 & 7.49 \\
\hline & $2009-8-2$ & 1 & 1.20 & RM3740 & RM3652 & 8.62 & -0.69 & 10.18 \\
\hline \multirow[t]{3}{*}{$q t l 2-1-3$} & $2009-2-1$ & 2 & 44.50 & RM5699 & RM5789 & 9.80 & -1.33 & 12.17 \\
\hline & Hoku1 & 2 & 44.50 & RM5699 & RM5789 & 3.31 & -0.94 & 8.43 \\
\hline & $2009-5-2$ & 2 & 44.50 & RM5699 & RM5789 & 8.78 & -0.82 & 14.30 \\
\hline \multirow[t]{8}{*}{ qtl9-1-8 } & $2009-5-1$ & 9 & 1.25 & RM219 & RM7390 & 9.25 & -0.52 & 10.34 \\
\hline & $2009-15$ & 9 & 1.25 & RM219 & RM7390 & 4.76 & -0.44 & 9.54 \\
\hline & $2009-2-1$ & 9 & 1.25 & RM219 & RM7390 & 6.20 & -0.46 & 11.29 \\
\hline & 2009-11-1 & 9 & 1.25 & RM219 & RM7390 & 3.76 & -0.30 & 7.43 \\
\hline & $2009-10$ & 9 & 1.25 & RM219 & RM7390 & 6.90 & -0.58 & 13.25 \\
\hline & Hoku1 & 9 & 1.25 & RM219 & RM7390 & 4.38 & -0.42 & 10.09 \\
\hline & $2009-8-2$ & 9 & 12.01 & RM219 & RM7390 & 8.62 & -0.69 & 15.18 \\
\hline & $2009-9$ & 9 & 33.01 & RM219 & RM7390 & 5.07 & -0.61 & 14.22 \\
\hline qtl9-2-1 & $2009-7$ & 9 & 100.10 & RM215 & RM6707 & 14.57 & -1.78 & 28.62 \\
\hline qtll1-1-1 & $2009-8-2$ & 11 & 0.05 & RM3668 & RM332 & 14.95 & -1.72 & 15.94 \\
\hline \multirow[t]{3}{*}{ qtll1-2-3 } & $2009-12-3$ & 11 & 21.50 & RM167 & RM3701 & 12.56 & -1.12 & 11.19 \\
\hline & Hoku1 & 11 & 29.20 & RM167 & RM3701 & 5.75 & -0.60 & 10.99 \\
\hline & $2009-2-1$ & 11 & 40.20 & RM167 & RM3701 & 4.50 & -0.54 & 12.46 \\
\hline \multirow[t]{7}{*}{ qtll1-3-7 } & $2009-15$ & 11 & 67.10 & RM3428 & RM6091 & 15.50 & -0.55 & 18.44 \\
\hline & $2009-10$ & 11 & 67.10 & RM3428 & RM6091 & 23.15 & -1.12 & 44.34 \\
\hline & $2009-7$ & 11 & 67.10 & RM3428 & RM6091 & 12.78 & -0.82 & 14.30 \\
\hline & Hoku1 & 11 & 67.10 & RM3428 & RM6091 & 13.31 & -0.62 & 14.34 \\
\hline & $2009-9$ & 11 & 67.10 & RM3428 & RM6091 & 8.95 & -0.63 & 13.58 \\
\hline & $2009-8-2$ & 11 & 67.10 & RM3428 & RM6091 & 20.44 & -1.88 & 14.29 \\
\hline & $2009-13$ & 11 & 67.10 & RM3428 & RM6091 & 6.58 & -0.93 & 10.97 \\
\hline \multirow[t]{9}{*}{ qtll1-4-9 } & $2009-5-1$ & 11 & 72.40 & RM6091 & RM26632 & 15.29 & -0.70 & 21.09 \\
\hline & $2009-15$ & 11 & 72.40 & RM6091 & RM26632 & 6.47 & -0.64 & 17.77 \\
\hline & $2009-12-3$ & 11 & 72.40 & RM6091 & RM26632 & 28.15 & -1.96 & 80.82 \\
\hline & $2009-10$ & 11 & 72.40 & RM6091 & RM26632 & 3.13 & -0.25 & 9.90 \\
\hline & $2009-7$ & 11 & 72.40 & RM6091 & RM26632 & 18.03 & -1.19 & 25.15 \\
\hline & Hoku1 & 11 & 72.40 & RM6091 & RM26632 & 22.15 & -0.46 & 46.49 \\
\hline & $2009-5-2$ & 11 & 72.40 & RM6091 & RM26632 & 12.81 & -1.28 & 16.88 \\
\hline & $2009-8-2$ & 11 & 72.40 & RM6091 & RM26632 & 8.03 & -1.19 & 25.15 \\
\hline & $2009-13$ & 11 & 72.40 & RM6091 & RM26632 & 9.80 & -1.33 & 32.17 \\
\hline \multirow{4}{*}{ qtll1-5-4 } & $2009-2-1$ & 11 & 77.00 & RM26632 & RM4601 & 13.65 & -0.47 & 19.40 \\
\hline & $2009-11-1$ & 11 & 77.00 & RM26632 & RM4601 & 8.92 & -0.50 & 11.46 \\
\hline & $2009-10$ & 11 & 77.00 & RM26632 & RM4601 & 3.21 & -0.27 & 10.65 \\
\hline & $2009-9$ & 11 & 77.00 & RM26632 & RM4601 & 5.74 & -0.60 & 12.12 \\
\hline \multirow[t]{4}{*}{ qtll1-6-4 } & $2009-5-1$ & 11 & 139.10 & RM27291 & RM7654 & 12.16 & -0.94 & 10.43 \\
\hline & 2009-15 & 11 & 139.10 & RM27291 & RM7654 & 3.08 & -0.80 & 6.91 \\
\hline & Hoku1 & 11 & 139.10 & RM27291 & RM7654 & 5.29 & -0.70 & 11.09 \\
\hline & $2009-5-2$ & 11 & 139.10 & RM27291 & RM7654 & 15.03 & -1.17 & 18.47 \\
\hline \multirow[t]{3}{*}{ qtll1-7-3 } & $2009-5-2$ & 11 & 141.91 & RM27341 & RM27371 & 15.65 & -1.19 & 12.81 \\
\hline & $2009-9$ & 11 & 141.91 & RM27341 & RM27371 & 5.75 & -0.59 & 13.29 \\
\hline & 2009-13 & 11 & 141.91 & RM27341 & RM27371 & 9.19 & -0.86 & 13.37 \\
\hline qtll2-1-1 & $2009-12-3$ & 12 & 0.25 & RM2851 & RM6953 & 6.98 & -0.68 & 2.40 \\
\hline \multirow[t]{3}{*}{ qtll2-2-3 } & $2009-12-3$ & 12 & 28.20 & RM7619 & RM27618 & 13.30 & -1.35 & 12.98 \\
\hline & $2009-7$ & 12 & 30.20 & RM7619 & RM27618 & 8.62 & -0.69 & 15.81 \\
\hline & $2009-9$ & 12 & 30.20 & RM7619 & RM27618 & 7.90 & -0.69 & 11.68 \\
\hline
\end{tabular}

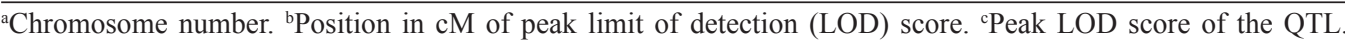
${ }^{\mathrm{d}}$ Additive effect explained at peak LOD score. ${ }^{\circ}$ Explained the contribution to the phenotypic variance. 
Chr 1

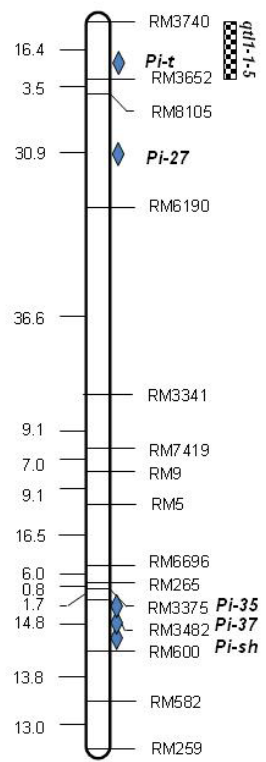

Chr 11

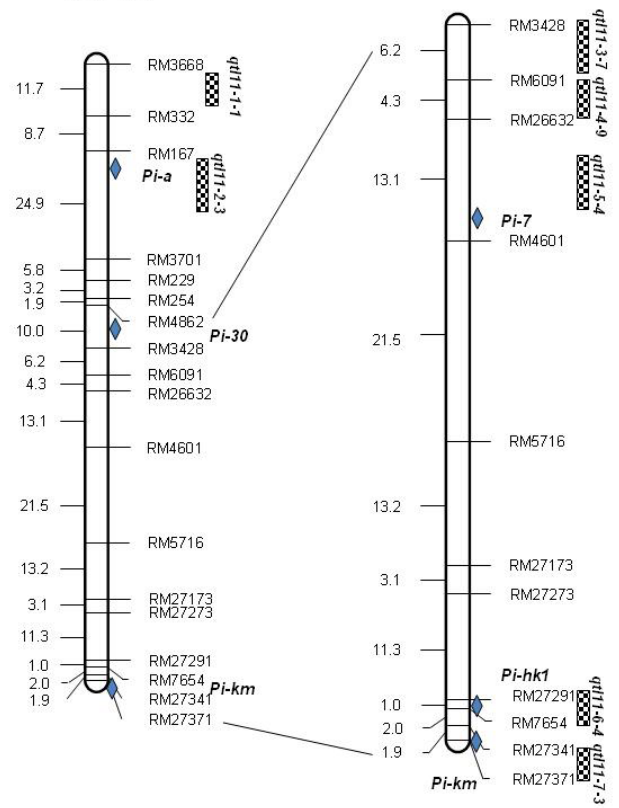

Chr 9

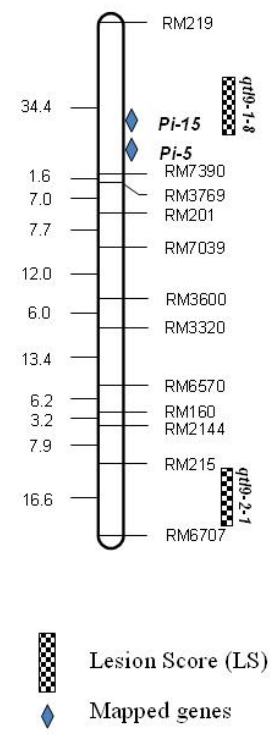

Chr 12

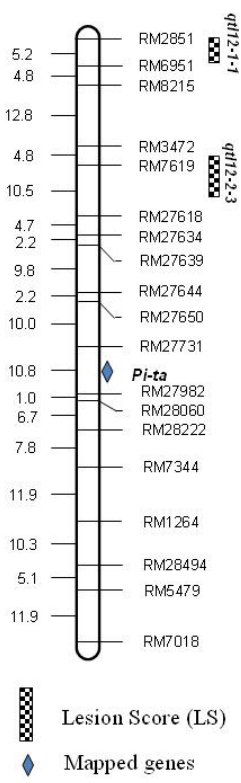

Figure 2. Genetic locations of quantitative trait loci (QTL) for resistance to blast detected based on lesion score. The locations of cloned resiatance genes were collected from China Rice Data Centre (http://www.ricedata.cn) and the paper by Liu et al. (2010). The SSR markers, mapped resistance genes, and QTL are located on the right side of the chromosomes. The distance between two SSR markers is located on the left side of the chromosomes. 
Among the 7 QTL on chromosome 11, qtl11-4-9 was effective against 9 isolates, including 2009-5-1(ZA7), 2009-15(ZB13), 2009-12-3(ZC3), 2009-10(ZF1), 2009-7(ZG1), Hoku1(ZG1), 2009-5-2(ZA7), 2009-8-2(ZC13), and 2009-13(ZD5), with LOD scores of 3.1328.15 and phenotypic variances of 9.90-80.82\%. The QTL qtl11-3-7 was effective against 7 isolates, including 2009-15(ZB13), 2009-10(ZF1), 2009-7(ZG1), Hoku1(ZG1), 20099(ZB15), 2009-8-2(ZC13), and 2009-13(ZD5), with LOD scores of 6.58-23.15 and phenotypic variances of 10.97-44.34\%. The QTL qtl11-5-4 was effective against 4 isolates, including 2009-2-1(ZD7), 2009-11-1(ZE3), 2009-10(ZF1), and 2009-9(ZB15), with LOD scores of 3.21-13.65 and phenotypic variances of 10.65-19.40\%. The other 4 QTL, qtl11-6-4, qtl11-2-3, qtl11-7-3, and qtl11-1-1, were effective against 4, 3, 3, and 1 isolates, respectively.

Among the 2 QTL on chromosome 9, qtl9-1-8 was effective against 8 isolates, including 2009-15(ZB13), 2009-2-1(ZD7), 2009-11-1(ZE3), 2009-10(ZF1), Hoku1(ZG1), 2009-51(ZA7), 2009-8-2(ZC13), and 2009-9(ZB15), with LOD scores of 3.76-9.25 and phenotypic variances of 7.43-15.18\%. qtl9-2-1 was only effective against 1 isolate, 2009-7(ZG1), with an LOD score of 14.57 and phenotypic variance of $28.62 \%$. Among the 2 QTL on chromosome 12, qtll2-2-3 was effective against 3 isolates, including 2009-12-3(ZC3), 2009-7(ZG1), and 2009-9(ZB15), yielding LOD scores of 7.90-13.30 and phenotypic variances of 11.6815.81\%. qtl12-1-1 was effective against 1 isolate 2009-12-3(ZC3) with an LOD score of 6.98 and phenotypic variance of 2.4 .

The qtl1-1-5, which was anchored on chromosome 1, was effective against 5 isolates, including 2009-11-1(ZE3), 2009-10(ZF1), 2009-7(ZG1), Hoku1(ZG1), and 2009-8-2 (ZC13), with LOD scores of 3.52-8.62 and phenotypic variances of 7.49-13.35\%. qtl2-1-3, which was detected on chromosome 2, was effective against 3 isolates, including 2009-2-1(ZD7), Hoku1(ZG1), and 2009-5-2(ZA7), with LOD scores of 3.31-9.80 and phenotypic variances of $8.43-14.30 \%$.

\section{Properties of 5 newly identified resistance QTL}

Among the 13 QTL, 5 QTL were not reported previously, including 3 QTL, qtl111-1, qtl11-3-7, qtl11-4-9, anchored on chromosome 11, and 2, qtl12-1-1 and qtl12-2-3, on chromosome 12. qtl11-3-7 and qtl11-4-9 may be the 2 main effective QTL and show resistance to 7 and 9 isolates, respectively. The former presented a maximum LOD score of 23.15 and phenotypic variance of $44.34 \%$, while the latter provided a maximum LOD score of 28.15 and phenotypic variance of $80.82 \%$. qtl11-1-1, qtl12-1-1, and qtl12-2-3 were also newly identified QTL, and showed LOD scores and phenotypic variances of 14.95 and 15.94\%, 6.98 and $2.40 \%$, and 13.30 and $12.98 \%$ (maximum), respectively.

\section{DISCUSSION}

In the present study, Bodao was resistant to 12 blast isolates belonging to 7 races based on the Chinese Rice Blast Identification System. This result was consistent with those of a previous report (Li et al., 2007), which showed that the japonica landrace Bodao was broadly and highly resistant to rice blast. At least 5 landraces, including Bodao, Heikezijing, Tieganqing, Jiangnanwan, and Quernuo, were identified as highly resistant varieties from more than 2000 landraces collected from the Taihu Lake region (Li et al., 2007). In our previous study, 22 QTL 
of Heikezijing that conferred resistance to 19 blast isolates were mapped onto chromosomes 1, 7, 9, 11, and 12 via QTL detection (Shi et al., 2010). To effectively dissect resistance loci to Chinese isolates in Bodao, a population of 155 RILs $\left(\mathrm{F}_{2: 8}\right)$ was derived from a cross between Bodao and Suyunuo, a highly blast-susceptible landrace. Twelve isolates belonging to all 7 races were chosen to inoculate this population so that $13 \mathrm{QTL}$ on chromosomes 1,2, 9, 11, and 12 were detected from Bodao.

The QTL detection approach has been used to localize major or minor loci involved in rice blast resistance at the molecular level (Sallaud et al., 2003; Wu et al., 2005; Shi et al., 2010). In the present study, 13 QTL in Bodao were identified by QTL mapping and lesion score determination. The lesion score was typically considered to be the trait for QTL mapping (Sallaud et al., 2003; Shi et al., 2010). The reaction of rice to blast was scored according to a 6-class scale based on lesion type (Sallaud et al., 2003). Individuals with scores of 0, 1, and 2 were considered resistant, while those with scores of 3,4 , and 5 were considered susceptible. However, plants with critical scores of 2 or 3 were difficult to classify in the inheritance dissection of resistance. Through RIL populations derived from resistant $\mathrm{x}$ susceptible accession, partial resistance loci were detected by applying a QTL mapping method based on the reaction of each line to blast isolates. The percentages of the diseased leaf area, lesion number, and lesion size were used to identify QTL in Gumei 2, a durably resistant indica cultivar (Wu et al., 2005). The unique standards of these traits and an accurate evaluation system will be helpful for detecting partial resistance loci.

A total of 13 resistant QTL were detected from Bodao based on lesion scores. Five QTL in 5 distinct regions were first reported in this paper, including qtl11-1-1, qtl11-3-7, qtl114-9, qtl12-1-1, and qtl12-2-3. Among these QTL, qtl11-4-9, which presented LOD scores of 3.13-28.15 and phenotypic variances of 9.90-80.82\%, and qtl11-3-7, with LOD scores of 6.5823.15 and phenotypic variances of $10.97-44.34 \%$, are 2 new main effective QTL. We focused on the fine mapping and cloning of these 2 genes. Four QTL in 4 regions, including qtl1-1-5, $q t l 2-1-3, q t l 9-1-8$, and $q t l 11-7-3$, were close to Pi-t, Pi-d1, Pi-15, Pi-hkl, and Pi- $k^{m}$, respectively (Pan et al., 2003; Ashikawa et al., 2008; Hayashi and Yoshida, 2009; Liu et al., 2010). Three QTL in 3 regions, including qtl11-2-3, qtl11-5-4, and qtl11-6-4, were close to Pi-a, Pi-7, and $P i-h k 1$, respectively (Inukai et al., 1996; Shi et al., 2010; Okuyama et al., 2011). Further, fine mapping results and allelism tests between reference cultivars with known resistance genes are necessary to determine whether these 7 QTL are new resistance gene alleles.

In summary, we mapped rice blast-resistance QTL based on lesion scores using 155 RILs from a cross of Bodao x Suyunuo. Moreover, we determined that qtl11-3-7 was effective against 7 isolates and qtl11-4-9 was effective against 9 isolates using QTL mapping. The information obtained in the present study will be valuable for further studies examining map-based cloning resistance genes or breeding blast resistance in rice through molecularassistant selection.

\section{ACKNOWLEDGMENTS}

Research supported by grants from the National Key Project for Transgenic Crops (\#2011ZX08009-003, \#2014ZX08009-001B), the Natural Science Foundation of China (\#31171516) and the Fundamental Research Funds for the Central Universities (\#KYZ201302). We would like to thank Prof. Chen Zhi-Yi and Dr. Liu Yong-Feng for kindly providing all of the blast isolates. 


\section{REFERENCES}

Ashikawa I, Hayashi N, Yamane H, Kanamori H, et al. (2008). Two adjacent nucleotide-binding site-leucine-rich repeat class genes are required to confer Pikm-specific rice blast resistance. Genetics 180: 2267-2276.

Bryan GT, Wu KS, Farrall L, Jia Y, et al. (2000). A single amino acid difference distinguishes resistant and susceptible alleles of the rice blast resistance gene Pi-ta. Plant Cell 12: 2033-2046.

Chen X, Shang J, Chen D, Lei C, et al. (2006). A B-lectin receptor kinase gene conferring rice blast resistance. Plant J. 46: 794-804.

Dean RA, Talbot NJ, Ebbole DJ, Farman ML, et al. (2005). The genome sequence of the rice blast fungus Magnaporthe grisea. Nature 434: 980-986.

Deng Y, Zhu X, Shen Y and He Z (2006). Genetic characterization and fine mapping of the blast resistance locus Pigm $(t)$ tightly linked to $P i 2$ and $P i 9$ in a broad-spectrum resistant Chinese variety. Theor. Appl. Genet. 113: 705-713.

Fukuoka S, Saka N, Koga H, Ono K, et al. (2009). Loss of function of a proline-containing protein confers durable disease resistance in rice. Science 325: 998-1001.

Hayashi K and Yoshida H (2009). Refunctionalization of the ancient rice blast disease resistance gene Pit by the recruitment of a retrotransposon as a promoter. Plant J. 57: 413-425.

Hua L, Wu J, Chen C, Wu W, et al. (2012). The isolation of Pil, an allele at the Pik locus which confers broad spectrum resistance to rice blast. Theor. Appl. Genet. 125: 1047-1055.

Hulbert SH, Webb CA, Smith SM and Sun Q (2001). Resistance gene complexes: evolution and utilization. Annu. Rev. Phytopathol. 39: 285-312.

Inukai T, Zeigler RS, Sarkarung S, Bronson M, et al. (1996). Development of pre-isogenic lines for rice blast-resistance by marker-aided selection from a recombinant inbred population. Theor. Appl. Genet. 93: 560-567.

Jeung JU, Kim BR, Cho YC, Han SS, et al. (2007). A novel gene, Pi40(t), linked to the DNA markers derived from NBSLRR motifs confers broad spectrum of blast resistance in rice. Theor. Appl. Genet. 115: 1163-1177.

Kosambi DD (1944). The estimation of map distance from recombination values. Ann. Eugen. 12: 172-175.

Lander E and Kruglyak L (1995). Genetic dissection of complex traits: guidelines for interpreting and reporting linkage results. Nat. Genet. 11: 241-247.

Li PF, Shi XL, Wang JF and Zhang HS (2007). Genetic analysis of resistance to rice blast in four japonica landraces from Taihu Lake region. Yi Chuan 29: 1249-1255.

Ling ZZ, Lei CL and Wang JL (2004). Review and prospect for study of physiologic races on rice blast fungus (Pyricularia grisea). Sci. Agric. Sin. 37: 1849-1859.

Liu J, Wang X, Mitchell T, Hu Y, et al. (2010). Recent progress and understanding of the molecular mechanisms of the rice-Magnaporthe oryzae interaction. Mol. Plant Pathol. 11: 419-427.

Liu RH and Meng JL (2003). MapDraw: a microsoft excel macro for drawing genetic linkage maps based on given genetic linkage data. Yi Chuan 25: 317-321.

Mackill DJ and Bonman JM (1992). Inheritance of blast resistance in near-isogenic lines of rice. Phytopathology 82: 746-749.

Marcel TC, Varshney RK, Barbieri M, Jafary H, et al. (2007). A high-density consensus map of barley to compare the distribution of QTL for partial resistance to Puccinia hordei and of defence gene homologues. Theor. Appl. Genet. 114: 487-500.

Okuyama Y, Kanzaki H, Abe A, Yoshida K, et al. (2011). A multifaceted genomics approach allows the isolation of the rice Pia-blast resistance gene consisting of two adjacent NBS-LRR protein genes. Plant J. 66: 467-479.

Ou SH (1979). Breeding Rice for Resistance to Blast, A Critical View. In: Proceedings of the Rice Blast Workshop. International Rice Research Institute, Manila, 79-137.

Pan QH, Hu ZD, Tanisaka T and Wang L (2003). Fine mapping of blast resistance gene Pi15, linked to Pii, on rice chromosome 9. Acta Bot. Sin. 45: 871-877.

Parlevliet JE (1979). Components of resistance that reduce the rate of epidemic development. Annu. Rev. Phytopathol. 17: 203-222.

Qu S, Liu G, Zhou B, Bellizzi M, et al. (2006). The broad-spectrum blast resistance gene Pi9 encodes a nucleotide-binding site-leucine-rich repeat protein and is a member of a multigene family in rice. Genetics 172: 1901-1914.

Rai AK, Kumar SP, Gupta SK, Gautam N, et al. (2011). Functional complementation of rice blast resistance gene Pi- $k^{h}$ (Pi54) conferring resistance to diverse strains of Magnaporthe oryzae. J. Plant Biochem. Biotechnol. 20: 55-65.

Sallaud C, Lorieux M, Roumen E, Tharreau D, et al. (2003). Identification of five new blast resistance genes in the highly blast-resistant rice variety IR64 using a QTL mapping strategy. Theor. Appl. Genet. 106: 794-803.

Shi X, Wang J, Bao Y, Li P, et al. (2010). Identification of the quantitative trait loci in japonica rice landrace Heikezijing 
responsible for broad-spectrum resistance to rice blast. Phytopathology 100: 822-829.

Vergne E, Grand X, Ballini E, Chalvon V, et al. (2010). Preformed expression of defense is a hallmark of partial resistance to rice blast fungal pathogen Magnaporthe oryzae. BMC Plant Biol. 10: 206.

Wang JF, He XJ, Zhang HS and Chen ZY (2002). Genetic analysis of blast resistance in japonica rice landrace heikezijing from Taihu region. Yi Chuan Xue Bao 29: 803-807.

Wang S, Basten CJ and Zeng ZB (2007). Windows QTL Cartographer 2.5. Department of Statistics, North Carolina State University, Raleigh. Available at [http://statgen.ncsu.edu/qtlcart/WQTLCart.htm]. Accessed March 1, 2013.

Wu JL, Fan YY, Li DB, Zheng KL, et al. (2005). Genetic control of rice blast resistance in the durably resistant cultivar Gumei 2 against multiple isolates. Theor. Appl. Genet. 111: 50-56.

Yuan B, Zhai C, Wang W, Zeng X, et al. (2011). The Pik-p resistance to Magnaporthe oryzae in rice is mediated by a pair of closely linked CC-NBS-LRR genes. Theor. Appl. Genet. 122: 1017-1028.

Zeng ZB (1994). Precision mapping of quantitative trait loci. Genetics 136: 1457-1468.

Zhai C, Lin F, Dong Z, He X, et al. (2011). The isolation and characterization of Pik, a rice blast resistance gene which emerged after rice domestication. New Phytol. 189: 321-334.

Zhang H, Liu K, Zhang X, Tang W, et al. (2011). Two phosphodiesterase genes, PDEL and PDEH, regulate development and pathogenicity by modulating intracellular cyclic AMP levels in Magnaporthe oryzae. PLoS One 6: e17241.

Zhou B, Qu S, Liu G, Dolan M, et al. (2006). The eight amino-acid differences within three leucine-rich repeats between $\mathrm{Pi} 2$ and Piz-t resistance proteins determine the resistance specificity to Magnaporthe grisea. Mol. Plant Microbe Interact. 19: 1216-1228. 